
КРАНИОФАРИНГИОМЫ

(с Астафьева Л.И. ${ }^{1}$, Клочкова И.С. ${ }^{1}$, Калинин П.Л. ${ }^{1}$, Кадашев Б.А. ${ }^{1}$, Коновалов А.Н. ${ }^{1}$, Фомичев Д.В. ${ }^{1}$, Кутин М.А. ${ }^{\text {, }}$ Шарипов О.И. ${ }^{1}$, Сиднева Ю.Г. ${ }^{1}$ Яценко Д.А. ${ }^{2}$, Дзеранова Л.К. ${ }^{3}$

'ФГАУ «Национальный медицинский исследовательский центр нейрохирургии имени академика Н.Н. Бурденко»

Минздрава РФ, Москва

ФГБОУ ДПОРМАНПО Минздрава РФ

зФГБУ «Национальный медицинский исследовательский центр эндокринологии» Минздрава России, Москва

Ключевой структурой в регуляции энергетического гомеостаза является гипоталамус. Поражение гипоталамической области может приводить к дисбалансу энергообмена с развитием ожирения или кахексии. Наиболее часто обменные нарушения встречаются при краниофарингиомах. В статье представлены два клинических наблюдения папиллярной краниофарингиомы (КФ) у молодых женщин. Оба случая сопровождались нарушением энергетического баланса, в одном - с развитием ожирения, в другом - кахексии.

КЛЮЧЕВЫЕ СЛОВА: краниофарингиома, диэнцефальная кахексия, диэнцефальное ожирение, синдром Рассела, опухоль гипоталамуса, гипопитуитаризм, несахарный диабет.

\title{
OBESITY AND CAHEXIA AS THE FIRST MANIFESTATIONS OF CRANIOPHARINGIOMA
}

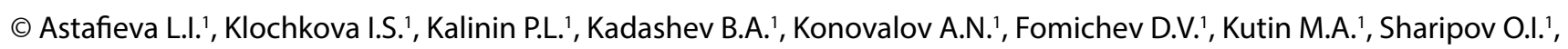
Sidneva Y.G. ${ }^{1}$, Yatsenko D.A. ${ }^{2}$, Dzeranova L.K. ${ }^{3}$

${ }^{1}$ N.N. Burdenko National Medical Research Center of Neurosurgery, Moscow, Russia

${ }^{2}$ FGBU RMANPO, Moscow, Russia

${ }^{3}$ Endocrinology Research Centre, Moscow, Russia

The key structure in the regulation of energy homeostasis is the hypothalamus. The damage of the hypothalamic region can lead to imbalance of energy exchange with the development of obesity or cachexia. The most common metabolic disorders occur in case of craniopharyngiomas. The article presents two clinical observations of papillary craniopharyngioma in young women. Cases were accompanied by different disturbances of the energy balance, in one - with the development of obesity, in the other - cachexia.

KEYWORDS: craniopharyngioma, diencephalic cachexia, diencephalic obesity, Russell's syndrome, hypothalamic tumor, hypopituitarism, diabetes insipidus.

\section{ВВЕДЕНИЕ}

Способность человека поддерживать стабильную массу тела на протяжении длительного времени обусловлена наличием механизмов точного контроля над поступлением и расходом энергии. Ключевой структурой в регуляции энергетического гомеостаза является гипоталамус. В настоящее время известно, что в пределах гипоталамуса существуют две группы нейронов, противоположно действующих на энергетический обмен [1-3]. Активность данных нейронов модулируется сигналами с периферии посредством гормонов, нейромедиаторов и нутриентов, что в конечном итоге определяет интенсивность метаболизма и пищевое поведение.

Поражение гипоталамической области, в том числе опухолевой природы, может приводить к дисбалансу энергообмена с развитием ожирения или кахексии. Наиболее часто обменные нарушения встречаются при краниофарингиомах (КФ) [4]. В статье представлены два клинических наблюдения КФ у молодых женщин. Оба случая сопровождались нарушением энергетического баланса, в одном - с развитием ожирения, в другом - кахексии.
Клинический случай 1

Пациентка Д., 36 лет, обратилась с жалобами на снижение зрения, увеличение веса с 65 до 71 кг в течение месяца, отсутствие менструаций.

Из анамнеза. Пациентка замужем, имеет здорового ребенка 16 лет от первого брака. Во втором браке в течение 10 лет не использовалась контрацепция, беременность не наступала. С июля 2016 г. развилась вторичная аменорея. С декабря 2016 г. пациентка стала отмечать увеличение веса и снижение зрения, что явилось поводом для обследования. На МРТ головного мозга обнаружена опухоль хиазмально-селлярной области. Амбулаторное обследование, проведенное в НМИЦН им. ак. Н.Н. Бурденко, позволило диагностировать преимущественно кистозную КФ эндо-супраселлярной локализации (рис. 3 А, Б); зрительные нарушения в виде битемпоральной гемианопсии и снижения остроты зрения (VIS OU=0,8); вторичные гипотиреоз и гипогонадизм, умеренную гиперпролактинемию. Пациентке было рекомендовано хирургическое лечение, которое планировалось провести через 2 мес. В течение последующих двух месяцев наблюдалось интенсивное увеличение веса с 71 
Таблица 1. Результаты гормонального и биохимического анализа крови двух пациенток до и после операции (пояснения в тексте)

\begin{tabular}{|c|c|c|c|c|c|}
\hline \multirow{3}{*}{ Tect } & \multicolumn{4}{|c|}{ Результат } & \multirow{3}{*}{$\begin{array}{c}\text { Референсный } \\
\text { интервал }\end{array}$} \\
\hline & \multicolumn{2}{|c|}{$\begin{array}{c}\text { женщина } 36 \text { лет } \\
\text { клинический случай } 1\end{array}$} & \multicolumn{2}{|c|}{$\begin{array}{c}\text { женщина } 24 \text { лет } \\
\text { клинический случай } 2\end{array}$} & \\
\hline & $\begin{array}{c}\text { до опера- } \\
\text { ции }\end{array}$ & $\begin{array}{l}\text { на } 7 \text { сутки по- } \\
\text { сле операции }\end{array}$ & $\begin{array}{c}\text { до опера- } \\
\text { ции }\end{array}$ & $\begin{array}{c}\text { на } 7 \text { сутки после } \\
\text { операции }\end{array}$ & \\
\hline \multicolumn{6}{|c|}{ Гормональный и биохимический анализы крови } \\
\hline Пролактин & $1051 * / 202$ & 156 & 4081 & 1005 & 59-619 мЕ/л \\
\hline Соматотропин & - & - & 23 & 2,01 & $<8,00$ нг/мл \\
\hline ИРФ-1 & 115 & 34,1 & 53,7 & 73,6 & $116,0-358,0$ нг/мл \\
\hline Кортизол & 306 & - & 329 & - & 119-618 нмоль/л \\
\hline Тиротропин (ТТГ) & 1,52 & 0,02 & 1,41 & 0,19 & 0,40-4,00 мЕ/л \\
\hline Свободный тироксин (Св.Т4) & $9,4^{* *} / 10,9$ & 10,0 & 10,7 & 8,2 & 11,5-22,7 пмоль/л \\
\hline Свободный трийодтиронин (Св.ТЗ) & 3,48 & 1,67 & 2,73 & 1,96 & 3,50-6,50 пмоль/л \\
\hline Лютропин (ЛГ) & 1,0 & $<0,1$ & $<0,1$ & - & $\begin{array}{l}\text { Ф. фаза 1,9-12,5 E/л } \\
\text { Л. фаза 0,5-16,9 E/л }\end{array}$ \\
\hline Фоллитропин (ФСГ) & 4,7 & 0,1 & 0,6 & - & $\begin{array}{c}\text { Ф. фаза 2,5-10,2 E/л } \\
\text { Л. фаза 1,5-9,1 Е/л }\end{array}$ \\
\hline Эстрадиол & 96 & 74 & 70 & - & $\begin{array}{c}\text { Ф. фаза } \\
\text { 72-529 пмоль/л } \\
\text { Л. фаза } \\
\text { 205-786 пмоль/л }\end{array}$ \\
\hline Лептин & 14,3 & - & 0,6 & - & 1,7-28 нг/мл \\
\hline Инсулин & 58 & - & 18 & - & 22-180 пмоль/л \\
\hline Паратгормон & 4,40 & - & 2,70 & - & 1,48-7,63 пмоль/л \\
\hline Гликированный гемоглобин (HbA $\left.{ }_{1 c}\right)$ & 4,3 & - & 4,7 & & $<6,1 \%$ \\
\hline Осмоляльность & 480 & - & 286 & - & $\begin{array}{c}275-295 \text { мосм/кг } \\
\mathrm{H}_{2} \mathrm{O}\end{array}$ \\
\hline Натрий & 143 & 151 & 143 & 149 & \\
\hline Калий & 4,5 & 4,1 & 4,2 & 4,0 & \\
\hline АЛТ & 78 & 43 & 80 & 380 & $>69$ ЕД/л \\
\hline $\mathrm{ACT}$ & 63 & 32 & 27 & 167 & $>46$ ЕД/л \\
\hline Альбумин & - & 33 & 32 & & $35-52$ г/л \\
\hline Триглицериды & 1,95 & - & 0,55 & - & $<2,25$ ммоль/л \\
\hline Холестерин & 5,95 & - & 2,46 & - & $<5,2$ ммоль/л \\
\hline ЛПВП & 0,56 & - & 1,18 & - & >1,04 ммоль/л \\
\hline лПнП & 4,5 & - & 1,0 & - & $<3,5$ ммоль/л \\
\hline Индекс атерогенности & 10,6 & - & 2,1 & - & $<5,2$ ед \\
\hline Кальций ионизированный & 1,19 & - & 1,17 & - & 1,09-1,30 ммоль/л \\
\hline Фосфор & 1,03 & - & 1,0 & - & 0,87-1,45 ммоль/л \\
\hline \multicolumn{6}{|c|}{ Клинический и биохимический анализ утренней порции мочи } \\
\hline Удельный вес & 1015 & - & 1004 & - & $1005-1028$ \\
\hline Осмоляльность & 480 & - & 649 & - & $>300 \mathrm{MOCM} / \mathrm{kr}$ \\
\hline
\end{tabular}

* ПРЛ до терапии каберголином; ** св T4 до терапии L-тироксином

до 90 кг. Явных нарушений пищевого поведения (полифагии, булимии) не отмечалось. До операции по поводу вторичного гипотиреоза пациентка постоянно получала терапию Л-тироксином 50 мкг в сутки; с целью нормализации пролактина - каберголин 0,25 мг в неделю.
При поступлении: рост - 175 см, вес - 90 кг, индекс массы тела (ИМТ) - $30 \mathrm{kг} / \mathrm{M}^{2}$ (норма 18-25). Подкожно-жировая клетчатка развита избыточно, с преобладанием в абдоминальной области. Объем талии 112 см (рис.1 А, Б). АД 110/70 мм рт. ст. ЧСС - 63 в минуту. От- 


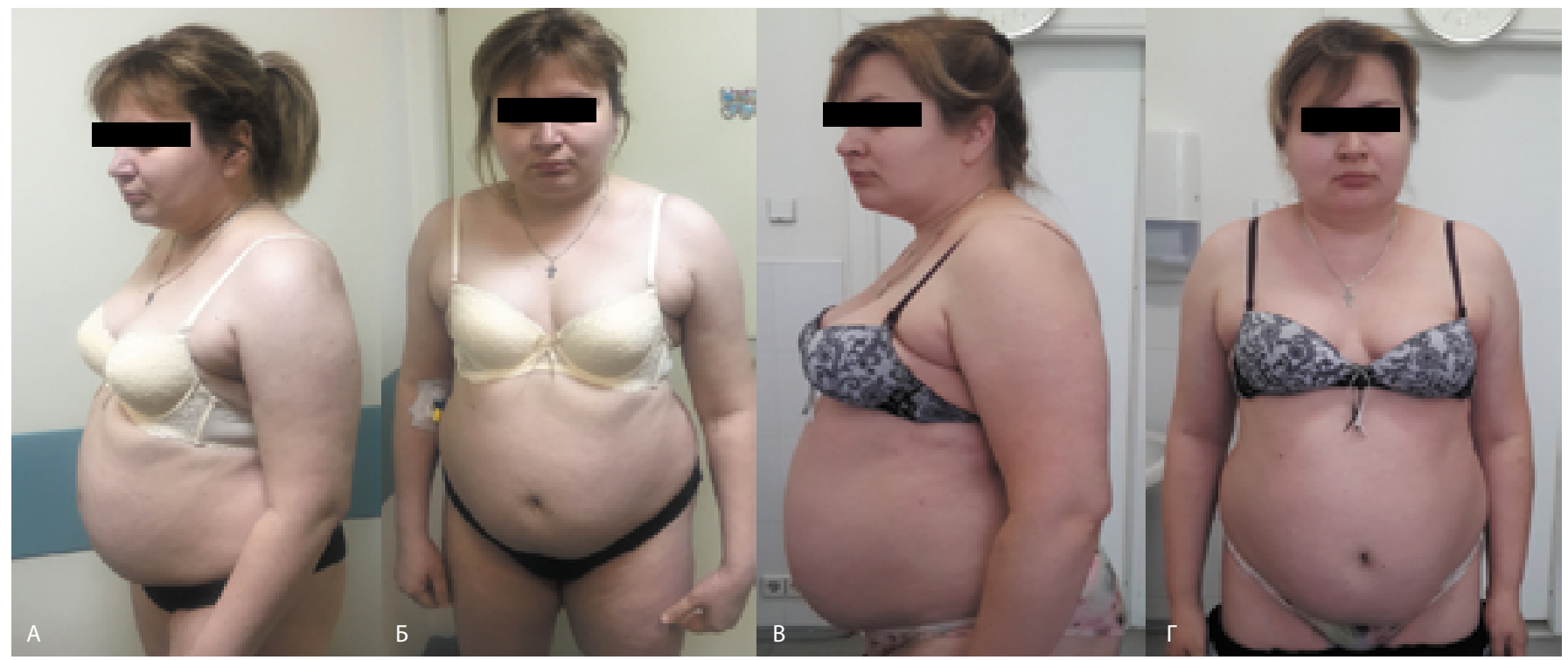

Рис. 1. Внешний вид пациентки Д. 36 лет.

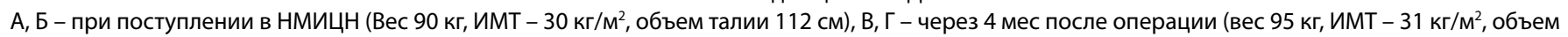
талии $118 \mathrm{cm)}$

мечено ухудшение зрительных функций: (VISOD=0,5; VISOS=0,2 битемпоральная гемианопсия). Данные лабораторных исследований представлены в табл. 1.

На основании клинических симптомов и результатов обследования диагностированы: стебельная КФ, хиазмальный синдром, диэнцефальное ожирение, вторичный гипогонадизм, вторичный гипотиреоз в стадии медикаментозной субкомпенсации; умеренная гиперпролактинемия, медикаментозно компенсированная.

Пациентке проведено удаление стебельной КФ из переднего расширенного транссфеноидального эндоскопического эндоназального доступа. Особенности протокола операции представлены в табл. 2.

Послеоперационный период осложнился развитием менингита, что потребовало проведения антибиотикотерапии в течение 14 дней. В послеоперационном периоде проводилась терапия гидрокортизоном (100 мг в сутки в/м в течение 7 дней, далее 25 мг в сутки перорально), доза Л-тироксина увеличена до 100 мкг перорально. На вторые сутки после операции развился несахарный диабет, что проявлялось в виде жажды и полиурии до 5200 мл/сут, гипернатриемии (натрий - 149151 ммоль/л). К терапии был добавлен таблетированный препарат десмопрессина. При нейроофтальмологическом контроле наблюдалось практически полное восстановление остроты зрения (VISOD=1,0; VISOS=0,9), грубые дефекты полей зрения с височных сторон сохранялись.

К моменту выписки (через 30 дней после операции) вес больной оставался без существенных изменений 90 кг, несмотря на соблюдение гипокалорийной диеты. Данными гормонального исследования был подтвержден пангипопитуитаризм (табл. 1). Пациентка была выписана с рекомендациями продолжить заместительную терапию вторичной надпочечниковой недостаточности (гидрокортизон 10 мг утром и 5 мг после обеда), вторичного гипотиреоза (Л-тироксин 100 мкг), несахарного диабета (десмопрессин по 0,2 мг 2 раза в день), а также гипогонадизма (фемостон). Кроме этого, было рекомендовано соблюдение низкокалорийной диеты (1000-1200 ккал/сут) и постепенное повышение ежедневной физической активности. В профилактических целях назначена терапия метформином в дозе 1500 мг в сутки.

Пациентка повторно обследована через 4 мес после хирургического лечения (рис. 1 В, Г). Она предъявляла жалобы на периодическое повышение температуры тела до $37,5^{\circ} \mathrm{C}$, увеличение веса на 5 кг, полиурию до 5000 мл/сут при пропуске приема препаратов десмопрессина. При осмотре: вес - 95 кг, объем талии 118 см, объем бедер 112 см. АД 110/70 мм рт. ст., ЧСС 76 в мин. Регулярно наблюдалась менструальноподобная реакция на фоне приема препарата фемостон. По клинико-биохимическим показателям достигнута компенсация питуитарной недостаточности. Уровни лептина и инсулина крови не превышали диапазона референсных значений (лептин - 19,6 нг/мл (1,7-28), инсулин - 9,1 мкЕд/мл (2,7-10,4)). Контрольная МРТ головного мозга с контрастированием не выявила явных остатков опухоли.

\section{Клинический случай 2}

Пациентка П., 24 лет обратилась с жалобами на прогрессирующее снижение веса, жажду, отсутствие менструального цикла, снижение зрения, головную боль.

Из анамнеза. Пациентка замужем, имеет двух здоровых детей 3 и 6 лет (две нормально протекающие беременности закончились срочными родами в 2009 и 2013 гг.). После вторых родов сохранялась аменорея. Однако пациентка не придала этому значения и к врачам не обращалась. В январе 2015 г. стала отмечать снижение веса на фоне адекватного питания. С марта 2015 г. появились жажда, снижение зрения, головная боль, снижение памяти. Постепенно потеряла в весе 20 кг (с 48 до 28 кг). В мае этого же года проведена МРТ головного мозга, на которой обнаружена опухоль хиазмально-селлярной области с распространением в третий (III) желудочек (рис. 3 В, Г).

При поступлении: рост - 161 см, вес - 28 кг, ИМТ 10,9 кг/м² (норма 18-25). Внешне выглядит старше своего возраста.Обращаетвнимание полное отсутствие подкожно-жировой клетчатки (рис. 2 А, Б, В). АД 80/60 мм рт. ст., ЧСС - 45 в минуту. Зубы и пальцы без признаков системного «рвотного» поведения. Выявлены зрительные нару- 
Таблица 2. Основные особенности роста опухолей, выявленные во время операции

\section{Пациентка Д. 36 лет \\ Клинический случай 1}

Доступ

Рост опухоли в III желудочек

Рост опухоли эндоселлярно

Состояние стебля гипофиза

Структура

Наличие крупных кист

Пересечение стебля гипофиза

Радикальность

Гистологический диагноз

шения в виде хиазмального синдрома. VISOD = 0,6; VISOS $=0,9$, битемпоральная гемианопсия. Данные лабораторных исследований представлены в табл. 1.

Пациентке поставлен диагноз: стебельная КФ, хиазмальный синдром, диэнцефальная кахексия, вторичный гипогонадизм, вторичный гипотиреоз, гиперпролактинемия.

02.09.2015 г. произведено транскраниальное удаление базальным лобно-височным доступом стебельной КФ (табл. 2). Сразу после операции проведена компьютерная томография головного мозга, которая подтвердила высокую степень радикальности операции, удаление опухоли с сохранением небольших остатков в инфундибулярной области III желудочка.

В послеоперационном периоде проводилась терапия гидрокортизоном (100 мг/сут в/м в течение 7 дней, далее 25 мг/сут перорально), препаратами гормонов щитовидной железы (Л-тироксин 25 мкг перорально). На вторые сутки после операции появились признаки несахарного диабета в виде жажды и полиурии до 4000 мл в сутки, гипернатриемии (натрий - 147-149 ммоль/л). К терапии был добавлен препарат десмопрессина 0,05 мг перорально 3 раза в день.

При нейроофтальмологическом контроле зрение сохранялось на дооперационном уровне. После операции пациентка стала активно набирать массу тела. К моменту выписки (через 12 дней после операции) пациентка прибавила в весе 14 кг, и её масса тела составила 42 кг
Пациентка П. 24 лет

Клинический случай 2

Транскраниальный базальный лобно-височный доступ

$+$

азрушен

плотная

$+$

$+$

субтотальная

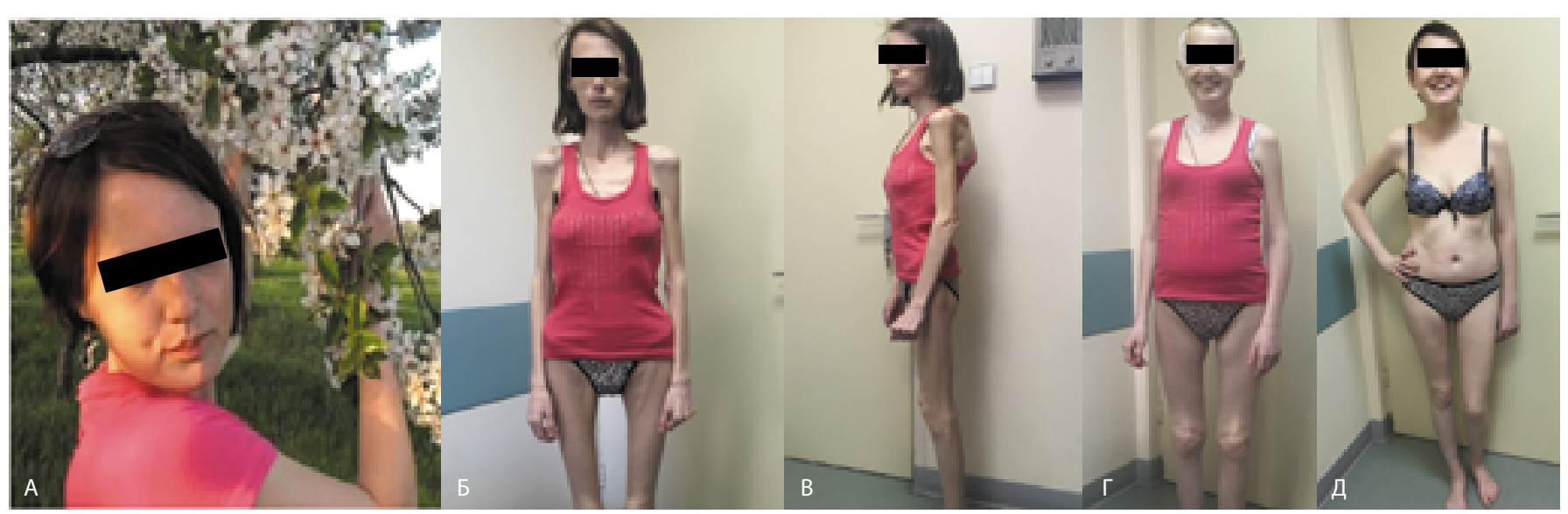

(рис. 2 Г). На основании гормонального обследования диагностирован пангипопитуитаризм, сохранялась умеренная гиперпролактинемия (пролактин-1005 мЕ/л) (табл. 1). Пациентка была выписана с рекомендациями продолжить заместительную терапию вторичной надпочечниковой недостаточности (Кортеф 10 мг утром и 5 мг после обеда), вторичного гипотиреоза (Л-тироксин 50 мкг), несахарного диабета (десмопрессин 0,1 мг 2 раза в день). В течение 3 следующих месяцев отмечалась прибавка массы тела еще на 4 кг с последующей стабилизацией веса в пределах 45-46 кг.

Контрольное обследование пациентки было проведено спустя 6 мес после операции. Масса тела 46 кг, ИМТ - 16,89 кг/м² (рис. 2 Д). Вторичная аменорея. При гормональном обследовании (18.01.2016) у пациентки сохранялись пангипопитуитаризм, несахарный диабет и повышение пролактина до 2884 МЕд/л (59-619). Отмечалась нормализация лептина - 5 нг/мл (1,7-28) и инсулина - 13 пмоль/л (22-180). Была проведена коррекция заместительной терапии, назначен препарат фемостон. При МРТ головного мозга с контрастированием выявлены остатки опухоли в области стебля гипофиза и дна III желудочка. Через 10 мес у пациентки диагностирован продолженный рост опухоли, проведено повторное транскраниальное удаление рецидива опухоли с последующим стереотаксическим облучением. За время последующего лечения вес пациентки значимо не менялся.

Рис. 2. Внешний вид пациентки П. 24 лет:

А - до начала проявления заболевания (вес 48 кг); Б, В - при поступлении в НМИЦН - практически отсутствует подкожно-жировая клетчатка, вес 28 кг; Г - через 12 дней после операции (вес с 28 кг увеличился до 42 кг); Д - через 6 мес после операции (вес 46 кг). 


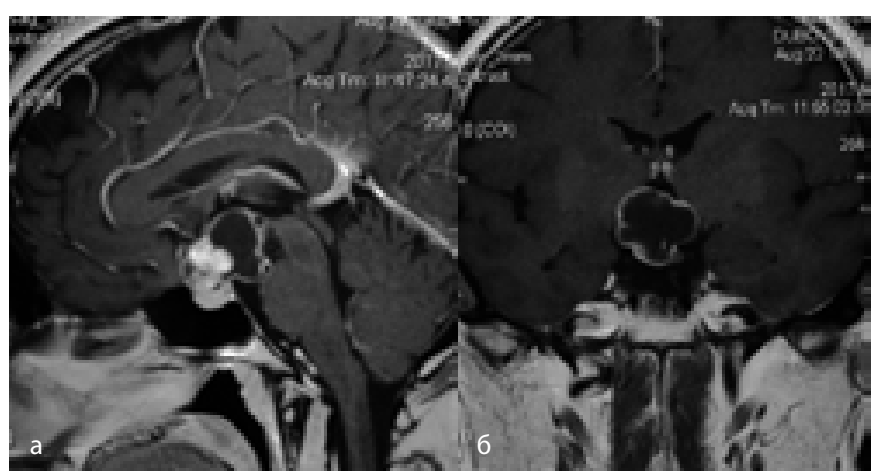

Рис. 3. МРТ пациентки Д. 36 лет с контрастным усилением в режиме Т1 в сагиттальной (а) и фронтальной (б) проекциях до операции.

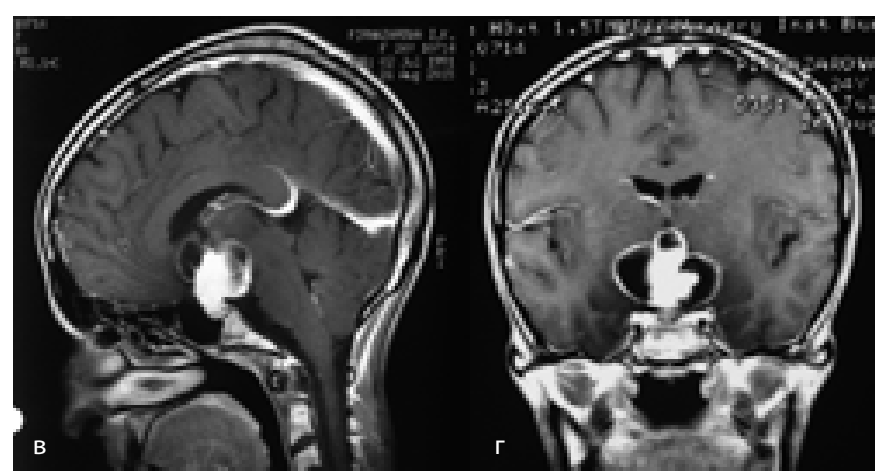

Рис. 3. МРТ пациентки П. 24 лет с контрастным усилением в режиме Т1 в сагиттальной (в) и фронтальной (г) проекциях до операции.

В обоих случаях визуализируются супраселлярные опухоли гетерогенной структуры с кистозными полостями и солидным компонентом, распространяющиеся в передние отделы III желудочка (картина стебельной краниофарингиомы)

\section{ОБСУЖДЕНИЕ}

КФ - доброкачественные эпителиальные опухоли (WHO I), которые развиваются из остатков эмбриональных клеток кармана Ратке, расположенного вдоль глоточно-гипофизарного хода. Частота КФ у взрослых составляет около 2,1-4,6\% всех интракраниальных новообразований [5-6]. Гистологически различают два основных типа КФ - адамантиноподобные и папилломатозные. КФ характеризуются наличием солидного и кистозного компонента, который выявляется примерно в $80 \%$ случаев [7]. Возникновение папилломатозной КФ, в отличие от адамантиноподобных, связывают с метаплазией эпителиальных клеток. Папилломатозные КФ встречаются, как правило, только у взрослых и локализуются преимущественно в области третьего (III) желудочка; одно из названий этих опухолей - папилломы III желудочка.

В силу своего анатомического расположения КФ, помимо гипопитуитаризма, часто вызывают развитие серьезных метаболических расстройств с развитием ожирения, реже - кахексии [8]. Согласно современным представлениям о центральной регуляции энергообмена, изменение массы тела объясняется дисфункцией гипоталамических нейронов вследствие их структурного повреждения. Патогенетические механизмы, лежащие в основе этих нарушений, сложны и до сих пор являются предметом научных исследований.

Известно, что КФ чаще ассоциированы с развитием гипоталамического (диэнцефального) ожирения (ГО) [9]. Оно выявляется в 30\% случаев на момент диагностики КФ, но чаще развивается вследствие ее хирургического лечения, достигая в некоторых сериях 60-85\% [10, 11]. Риск развития ГО после операции по поводу КФ определяется, в первую очередь, степенью вовлечения гипоталамуса в патологический процесс [12-15]. Результатом гипоталамического повреждения могут стать не только стремительное увеличение веса и развитие гиперфагии, но и вегетативная дисфункция, нарушение циркадных ритмов и терморегуляции, психические расстройства.

В первом клиническом наблюдении развитие ожирения у пациентки наблюдалось на фоне КФ до оперативного лечения. Быстрое нарастание массы тела протекало без явлений гиперфагии, на фоне адекватной коррекции вторичных гормональных нарушений (гипотиреоза, гиперпролактинемии). Именно быстрый темп увеличения веса, резистентный к модификации образа жизни, яв- ляется признаком, указывающим на гипоталамический уровень поражения. Интраоперационно было установлено, что опухоль имела гетерогенную структуру с формированием кист, достигала дна третьего желудочка, плотно срастаясь с его стенками. В послеоперационном периоде у пациентки сохранялось стойкое ожирение, несмотря на ограничение калорийности пищевого рациона до 1000 ккал/сут. Неэффективность общепринятой терапии ожирения, в том числе медикаментозной и бариатрической, объясняется низким уровнем метаболизма у пациентов с ГО, что было показано в ряде исследований [16-19]. При этом значимая роль отводится вегетативным нарушениям в виде снижения активности симпатоадреналовой системы и повышения тонуса блуждающего нерва с развитием гиперинсулинемии. Кроме того, аномалии сигнализации гормонов (лептина, инсулина, грелина) гипоталамическими нейронами, ввиду их резистентности к этим веществам, приводят к потере контроля над потреблением пищи (нарушению чувства голода и насыщения) и снижению расхода энергии [20]. Эти многочисленные нарушения определяют высокую сердечно-сосудистую заболеваемость и смертность среди данной группы пациентов [21, 22]. В настоящее время ГО остается серьезной медико-социальной проблемой, значимо ухудшающей качество жизни пациентов.

В отличие от ГО, диэнцефальная (гипоталамическая) кахексия (ДК) встречается крайне редко. Как правило, она наблюдается у детей первых 2-3 лет жизни, и лишь единичные случаи зарегистрированы у взрослых. Нами найдено описание только 4 случаев опухолевого поражения гипоталамуса с развитием ДК у взрослых - два из них были обусловлены КФ, два других - астроцитомой [23-26]. Развитие ДК наблюдается в основном на фоне глиом хиазмально-селлярной области разной степени злокачественности, сдавливающих дно третьего желудочка [27-29]. Другие типы опухолей, в том числе и КФ, как в нашем случае, скорее, являются исключением. Патофизиология ДК остается до сих пор неизученной. Наиболее вероятной причиной ее развития рассматривается чрезмерная активность популяции гипоталамических нейронов, обладающих катаболическим действием на основной обмен. Гипотеза об усилении метаболизма при ДК находит подтверждение в исследовании Vlachopapadopoulou E., в котором было показано увеличение расхода энергии на 30-50\% у пациентов с ДК по сравнению с группой контроля [30]. Следу- 
ет отметить, что прогрессирующая потеря веса протекает на фоне адекватного питания и удовлетворительного общего состояния пациентов. В нашем втором наблюдении пациентка сохраняла хорошее самочувствие и физическую активность, несмотря на выраженное истощение, что создавало контраст с ее внешним видом. Из лабораторных данных обращало внимание повышение базального уровня СТГ на фоне снижения ИРФ-1. Также выявлялись низкие уровни лептина и инсулина. Эти изменения свойственны ДК, но не являются причиной снижения массы тела, а развиваются вторично. Следует отметить, что отсутствие клинико-лабораторных данных гипокортицизма наглядно демонстрирует разные механизмы потери веса при ДК и синдромах Симмондса и Шиена. Сложно объяснить почему, но даже частичное удаление опухоли может привести к регрессу кахексии, что и произошло в нашем наблюдении. При отсутствии лечения большинство пациентов погибают в течение 1-2 лет [31].

Сравнивая представленные клинические наблюдения, хочется отметить, что на момент диагностики опухоли обе пациентки имели схожие гормональные нарушения в виде вторичного гипотиреоза и гипогонадизма. Также в обоих случаях до операции выявлялась сопутствующая гиперпролактинемия, более значимая у женщины с ДК. Это изменение, вероятнее, обусловлено блокадой дофаминергической системы при супраселлярной локализации опухоли. Других клинически значимых биохимических отклонений выявлено не было. Но особый интерес эти наблюдения представляют тем, что при сопоставимых характеристиках (размерах, топографии, структуры и морфологии (папиллярный тип КФ)) опухоли они оказывали абсолютно противоположное влияние на метаболизм. Существует предположение, что развитие ожирения на фоне КФ обусловлено инвазивным ее ростом по отношению к диэнцефальной области, а кахексии - компрессией опухолью сохранных гипоталамических структур [8]. Такое объяснение находит отражение и в представленных нами наблюдениях. Именно у женщины с кахексией метаболические нарушения имели обратимый характер. После операции она стала быстро прибавлять в весе, несмотря на наличие остатков опухоли в области III желудочка. Это указывает на решающую роль сдавления гипоталамуса в развитии кахексии у данной больной. При ГО, как показывают результаты экспериментальных работ и многочисленных исследований, основное значение отводится нарушению целостности гипоталамических ядер и их функциональных взаимосвязей с другими отделами головного мозга [8, 32-35].

К сожалению, расположение КФ приводит к нарастанию эндокринных расстройств после хирургического лечения [36, 37]. В обоих представленных наблюдениях после операции у пациенток появились дополнительные нарушения - несахарный диабет, центральный гипокортицизм, сохранились гипотиреоз и гипогонадизм (табл. 1). Обе женщины нуждаются в пожизненной заместительной терапии препаратами Л-тироксина, гидрокортизона, десмопрессина и половых стероидов.

\section{ЗАКЛЮЧЕНИЕ}

В представленных наблюдениях отображены особенности течения и трудности диагностики обменных нарушений, обусловленных КФ. Следует помнить, что изменения массы тела или другие эндокринные нарушения могут быть первым симптомом проявления этой опухоли. Детальное изучение механизмов, контролирующих энергообмен, может пролить свет на патогенез этих расстройств и определить целенаправленный подход в лечении пациентов с нарушением метаболизма.

\section{СПИСОК ЛИТЕРАТУРЫ | REFERENCES}

1. Kalra SP, Dube MG, Pu S, et al. Interacting Appetite-Regulating Pathways in the Hypothalamic Regulation of Body Weight1. Endocr. Rev. 1999;20(1):68-100. doi: 10.1210/edrv.20.1.0357

2. Austin J, Marks D. Hormonal Regulators of Appetite. Int. J. Pediatr. Endocrinol. 2009;2009:1-9. doi: 10.1155/2009/141753.

3. Sohn J-W. Network of hypothalamic neurons that control appetite. BMB Reports. 2015;48(4):229-233. doi: 10.5483/BMBRep.2015.48.4.272.

4. Castro DC, Cole SL, Berridge KC. Lateral hypothalamus, nucleus accumbens, and ventral pallidum roles in eating and hunger: interactions between homeostatic and reward circuitry. Front. Syst. Neurosci. 2015;9. doi: 10.3389/fnsys.2015.00090.

5. Sorva R, Heiskanen O, Perheentupa J. Craniopharyngioma in adults. Ann Clin. Res. 1987;19(5):339-343.

6. Bunin GR, Surawicz TS, Witman PA, et al. The descriptive epidemiology of craniopharyngioma. J. Neurosurg. 1998;89(4):547-551. doi: 10.3171/ jns.1998.89.4.0547.

7. Семенова Ж.Б. Гистобиология краниофарингиом и особенности течения заболевания. Дис. ... док. мед. наук. - Москва; 2000. [Semenova ZhB. Gistobiologiya kraniofaringiom i osobennosti techeniya zabolevaniya. [Dissertation] Moscow; 2000. (In Russ)] Доступно по: https://vivaldi.nlr. ru/bd000128590/details

8. Hoffmann A, Gebhardt U, Sterkenburg AS, et al. Diencephalic Syndrome in Childhood Craniopharyngioma-Results of German Multicenter Studies on 485 Long-term Survivors of Childhood Craniopharyngioma. J. Clin. Endocr. Metab. 2014;99(11):3972-3977. doi: 10.1210/jc.2014-1680

9. Müller HL, Emser A, Faldum A, et al. Longitudinal Study on Growth and Body Mass Index before and after Diagnosis of Childhood Craniopharyngioma. J. Clin. Endocr. Metab. 2004;89(7):3298-3305. doi: 10.1210/jc.2003-031751
10. 10. Muller HL, Gebhardt U, Teske C, et al. Post-operative hypothalamic lesions and obesity in childhood craniopharyngioma: results of the multinational prospective trial KRANIOPHARYNGEOM 2000 after 3-year follow-up. European journal of endocrinology / European Federation of Endocrine Societies. 2011;165(1):17-24. doi: 10.1530/eje-11-0158.

11. Daubenbüchel A, Müller H. Neuroendocrine Disorders in Pediatric Craniopharyngioma Patients. Journal of Clinical Medicine. 2015;4(3):389-413. doi: 10.3390/jcm4030389.

12. Roth $\mathrm{CL}$, Eslamy $\mathrm{H}$, Werny $\mathrm{D}$, et al. Semiquantitative analysis of hypothalamic damage on MRI predicts risk for hypothalamic obesity. Obesity. 2015;23(6):1226-1233. doi: 10.1002/oby.21067.

13. Van Gompel JJ, Nippoldt TB, Higgins DM, Meyer FB. Magnetic resonance imaging-graded hypothalamic compression in surgically treated adult craniopharyngiomas determining postoperative obesity. Neurosurg. Focus. 2010;28(4):E3. doi: 10.3171/2010.1.focus09303.

14. Müller HL. Craniopharyngioma and hypothalamic injury. Current Opinion in Endocrinology \& Diabetes and Obesity. 2016;23(1):81-89. doi: 10.1097/med.0000000000000214.

15. Elowe-Gruau E, Beltrand J, Brauner R, et al. Childhood Craniopharyngioma: Hypothalamus-Sparing Surgery Decreases the Risk of Obesity. J. Clin. Endocr. Metab. 2013;98(6):2376-2382. doi: 10.1210/jc.2012-3928.

16. Kim JH, Choi J-H. Pathophysiology and clinical characteristics of hypothalamic obesity in children and adolescents. Annals of Pediatric Endocrinology \& Metabolism. 2013;18(4):161. doi: 10.6065/apem.2013.18.4.161.

17. Roth CL. Hypothalamic Obesity in Patients with Craniopharyngioma: Profound Changes of Several Weight Regulatory Circuits. Front. Endocrinol. (Lausanne). 2011;2. doi: 10.3389/fendo.2011.00049.

18. Cohen M, Syme C, McCrindle BW, Hamilton J. Autonomic nervous system balance in children and adolescents with craniopharyngi- 
oma and hypothalamic obesity. European journal of endocrinology / European Federation of Endocrine Societies. 2013;168(6):845-852. doi: 10.1530/eje-12-1082.

19. Roth CL, Hunneman $\mathrm{DH}$, Gebhardt U, et al. Reduced Sympathetic Metabolites in Urine of Obese Patients With Craniopharyngioma. Pediatr. Res. 2007;61(4):496-501. doi: 10.1203/pdr.0b013e3180332cd6.

20. Holmer H, Pozarek G, Wirfält E, et al. Reduced Energy Expenditure and Impaired Feeding-Related Signals But Not High Energy Intake Reinforces Hypothalamic Obesity in Adults with Childhood Onset Craniopharyngioma. J. Clin. Endocr. Metab. 2010;95(12):5395-5402. doi: 10.1210/jc.2010-0993.

21. Olsson DS, Andersson E, Bryngelsson I-L, et al. Excess Mortality and Morbidity in Patients with Craniopharyngioma, Especially in Patients with Childhood Onset: A Population-Based Study in Sweden. J. Clin. Endocr. Metab. 2015;100(2):467-474. doi: 10.1210/jc.2014-3525

22. Erfurth EM, Holmer H, Fjalldal SB. Mortality and morbidity in adult craniopharyngioma. Pituitary. 2012;16(1):46-55. doi: 10.1007/s11102-012-0428-2.

23. Kohira I, Yoshimura Y, Ninomiya Y, et al. [Pilocytic astrocytoma and diencephalic syndrome in an adult with neurofibromatosis type 1]. Rinsho Shinkeigaku. 2003:43(6):327-329.

24. Tanabe M, WatanabeT, Hori T. Von Recklinghausen's disease with diencephalic syndrome in an adult. J. Neurosurg. 1994;80(3):556-558. doi: 10.3171/jns.1994.80.3.0556.

25. Sharma RR, Chandy MJ, Lad SD. Diencephalic syndrome of emaciation in an adult associated with a suprasellar craniopharyngioma-A case re port. Br. J. Neurosurg. 2009;4(1):77-80. doi: 10.3109/02688699009000687.

26. Miyoshi Y, Yunoki M, Yano A, Nishimoto K. Diencephalic Syndrome of Emaciation in an Adult Associated with a Third Ventricle Intrinsic Craniopharyngioma: Case Report. Neurosurgery. 2003;52(1):224-227. doi: 10.1097/00006123-200301000-00029.

27. 27. Stival A, Lucchesi M, Farina S, et al. An infant with hyperalertness, hyperkinesis, and failure to thrive: a rare diencephalic syndrome due to hypothalamic anaplastic astrocytoma. BMC Cancer. 2015;15(1). doi: 10.1186/s12885-015-1626-x
28. Rose C. Diencephalic Syndrome due to Hypothalamic Tumor: A Model of the Relationship between Weight and Puberty Onset. J. Clin. Endocr. Metab. 2006:91(7):2467-2473. doi: 10.1210/jc.2006-0322.

29. Brauner R, Trivin C, Zerah M, et al. Diencephalic Syndrome due to Hypothalamic Tumor: A Model of the Relationship between Weight and Puberty Onset. J. Clin. Endocr. Metab. 2006;91(7):2467-2473. doi: 10.1210/jc.2006-0322

30. Vlachopapadopoulou E, Tracey KS, Capella M, et al. Increased energy expenditure in a patient with diencephalic syndrome. The Journal of Pediatrics. 1993;122(6):922-924. doi: 10.1016/s0022-3476(09)90021-x.

31. Burr IM, Slonim AE, Danish RK, et al. Diencephalic syndrome revisited. The Journal of Pediatrics. 1976;88(3):439-444. doi: 10.1016/s0022-3476(76)80260-0.

32. Lustig RH. Hypothalamic Obesity after Craniopharyngioma: Mechanisms, Diagnosis, and Treatment. Front. Endocrinol. (Lausanne). 2011;2 doi: 10.3389/fendo.2011.00060.

33. Hetherington AW, Ranson SW. Hypothalamic lesions and adiposity in the rat. The Anatomical Record. 1940;78(2):149-172. doi: 10.1002/ar.1090780203

34. Daousi C, Dunn AJ, Foy PM, et al. Endocrine and neuroanatomic features associated with weight gain and obesity in adult patients with hypothalamic damage. The American Journal of Medicine. 2005:118(1):45-50. doi: 10.1016/j.amjmed.2004.06.035

35. Müller HL. Consequences of Craniopharyngioma Surgery in Children. J. Clin. Endocr. Metab. 2011;96(7):1981-1991. doi: 10.1210/jc.2011-0174.

36. Gorelyshev S., Matuev K., Lubnin A., et al. Brain tumors in infants: a report on 102 cases.// J Neuro-Oncology, Toronto, Ontario, Canada, June 24-27, 2012: Gorelyshev S, Matuev K, Lubnin A, et al., editors. Brain tumors in infants: a report on 103 cases. Neuro-oncology; 2012: OXFORD univ press inc journals dept, 2001 EVANS RD, CARY, NC 27513 USA.

37. Apuzzo ML, Levy ML, Tung H. Surgical Strategies and Technical Methodologies in Optimal Management of Craniopharyngioma and Masses Affecting the Third Ventricular Chamber. 1991;53:77-88. doi: 10.1007/978-3-7091-9183-5_14

\section{ИНФОРМАЦИЯ ОБ АВТОРАХ [AUTHORS INFO]}

Астафьева Людмила Игоревна, д.м.н.. [Liudmila I. Astafieva, Sc.D..]; адрес: Россия, 125047, Москва, улица 4-я Тверская-Ямская, д. 16 [address: 16 4-ya Tverskaya-Yamskaya street, 125047 Moscow, Russia]; ORCID: https://orcid.org/0000-0003-4480-1902; eLibrary SPIN: 4209-4723; e-mail: LAst@nsi.ru

Клочкова Ирина Сергеевна [lrina S.Klochkova]; ORCID: https://orcid.org/0000-0002-7440-4687; eLibrarySPIN: 8313-0190; e-mail: IKlochkova@nsi.ru. Калинин Павел Львович, д.м.н. [Pavel L. Kalinin, Sc.D.]; ORCID: https://orcid.org/0000-0001-9333-9473; eLibrarySPIN: 2336-5946; e-mail: pkalinin@nsi.ru. Кадашев Борис Александрович, А.М.Н., проф. [Boris A. Kadashev, Sc.D., Prof.]; ORCID: https://orcid.org/0000-0001-8344-3381; eLibrarySPIN: 4157-6654; e-mail: Kadashev@nsi.ru. Коновалов Александр Николаевич, д.М.Н., проф., акад. РАН [Aleksandr N. Konovalov, Sc.D., prof., academician of RAS]; eLibrarySPIN: 1462-1783; e-mail: AKonovalov@nsi.ru. Фoмичев Дмитрий Владиславович, к.м.н. [Dmitrii V. Fomichev, PhD]; ORCID: https://orcid.org/0000-0002-5323-1000; eLibrarySPIN: 3516-3513; e-mail: dfomichev@nsi.ru. Кутин Максим Александрович, к.м.н. [Maksim A. Kutin, PhD]; ORCID: https://orcid.org/0000-0002-6520-4296; eLibrarySPIN: 2336-5946; e-mail: kutin@nsi.ru. Waрипов Олег Ильдарович, К.М.H. [Oleg I. Sharipov, PhD]; ORCID: https://orcid.org/0000-0003-3777-5662; eLibrarySPIN: 3279-0844; e-mail: osharipov@nsi.ru. Сиднева Юлия Геннадьевна., к.м.н. [Ylia G. Sidneva, PhD]; ORCID: https://orcid.org/0000-0003-2733-5874; eLibrarySPIN: 5128-9059; e-mail: ysidneva@nsi.ru. Яценко Дина Александровна, врач [Dina A. Yatsenko, MD] ORCID: https://orcid.org/0000-0001-7726-2242; eLibrary SPIN:60559789, e-mail:dinochka-d@mail.ru. Дзеранова Лариса Константиновна, д.м.н. [Larisa K. Dzeranova, Sc.D.]; ORCID: https://orcid.org/0000-0002-0327-4619 eLibrary SPIN: 2958-5555; e-mail: dzeranovalk@yandex.ru

\section{ЦИТИРОВАТЬ:}

Астафьева Л.И., Клочкова И.С., Калинин П.Л., Кадашев Б.А., Коновалов А.Н., Фомичев Д.В., Кутин М.А., Шарипов О.И., Сиднева Ю.Г., Яценко Д.А., Дзеранова Л.К.. Ожирение и кахексия в качестве первых проявлений папиллярной краниофарингиомы. // Ожирение и метаболизм. - 2018. — Т.15. — №. 1 - C.43-49. doi: 10.14341/OMET2018143-49

\section{TO CITE THIS ARTICLE:}

Astafi eva LI, Klochkova IS, Kalinin PL, Kadashev BA, Konovalov AN, Fomichev DV, Kutin MA, Sharipov OI, Sidneva YG, Yatsenko D.A., Dzeranova LK. Obesity and cahexia as the first manifestations of craniopharingioma. Obesity and metabolism. 2018;15(1):43-49. doi: 10.14341/OMET2018143-49 